Supplement of E\&G Quaternary Sci. J., 69, 139-160, 2020

https://doi.org/10.5194/egqsj-69-139-2020-supplement

(c) Author(s) 2020. This work is distributed under

the Creative Commons Attribution 4.0 License.

(c) (1)

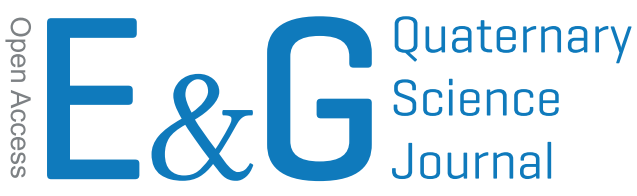

Supplement of

\title{
A 2600-year high-resolution climate record from Lake Trichonida (SW Greece)
}

Joana Seguin et al.

Correspondence to: Joana Seguin (jseguin@ecology.uni-kiel.de)

The copyright of individual parts of the supplement might differ from the CC BY 4.0 License. 


\section{$1 \quad$ Supplementary Online Material}

2 Table S1: Masterscale TRI1 indicating the respective sections used for the composite core 3 composition.

4 Fig. S1: Age-depth graph indicating all measured radiocarbon ages. Samples taken from bulk sediment (in red) consequently yield too old ages.

6 Fig. S2: Age-depth model on macroremain radiocarbon ages considering sedimentary units $11,12,14,16$, and 18 as deposited at once during a single e. g. turbidite event.

Fig. S3: Comparison graph with different age-depth models showing continuous sedimentation model (red) vs. event layer models (green, purple).

Fig. S4: Intensity diagrams of representative x-ray diffraction samples from different core sections. They show the presence of the main recognized minerals. CM: Clay minerals, Qz: Quartz, Ca: Calcite, Ar: Aragonite. 


\begin{tabular}{|c|c|c|c|c|c|}
\hline Core & Segment & $\begin{array}{l}\text { Masterscale top } \\
{[\mathrm{cm}]}\end{array}$ & $\begin{array}{l}\text { Masterscale bottom } \\
{[\mathrm{cm}]}\end{array}$ & $\begin{array}{l}\text { Section length } \\
{[\mathrm{cm}]}\end{array}$ & explanation for anchor point \\
\hline TRI1 A & 1 & 0 & 86 & 86 & thin dark gray layer in unit 17 in both cores \\
\hline TRI1 B & 1 & 86 & 140 & 54 & defined \\
\hline TRI1 A & 2 & 140 & 189 & 49 & upper boundary of dark unit 16 \\
\hline TRI1 B & 2 & 189 & 225 & 36 & defined \\
\hline TRI1 A & 3 & 225 & 256 & 31 & lower boundary of reddish unit 14 \\
\hline TRI1 B & 3 & 256 & 315 & 59 & lower boundary of reddish unit 11 \\
\hline TRI1 A & 4 & 315 & 360 & 45 & defined \\
\hline TRI1 B & 4 & 360 & 438.5 & 78.5 & end of core segment \\
\hline
\end{tabular}

Table S1 

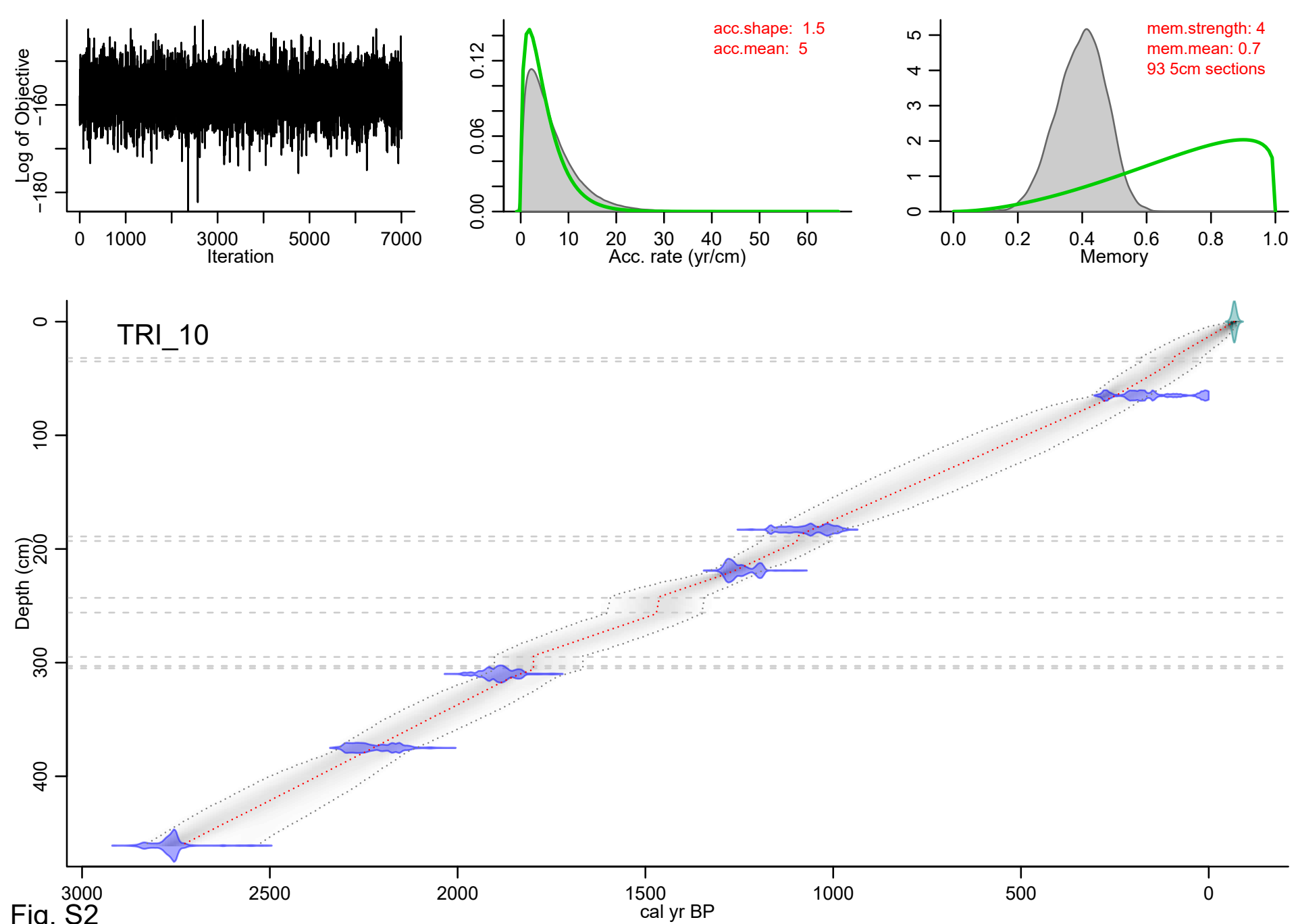

Fig. S2

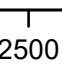

2000

cal yr BP 
$\frac{\text { E }}{\frac{E}{0}}$

300 -

400 -

Fig. S3 

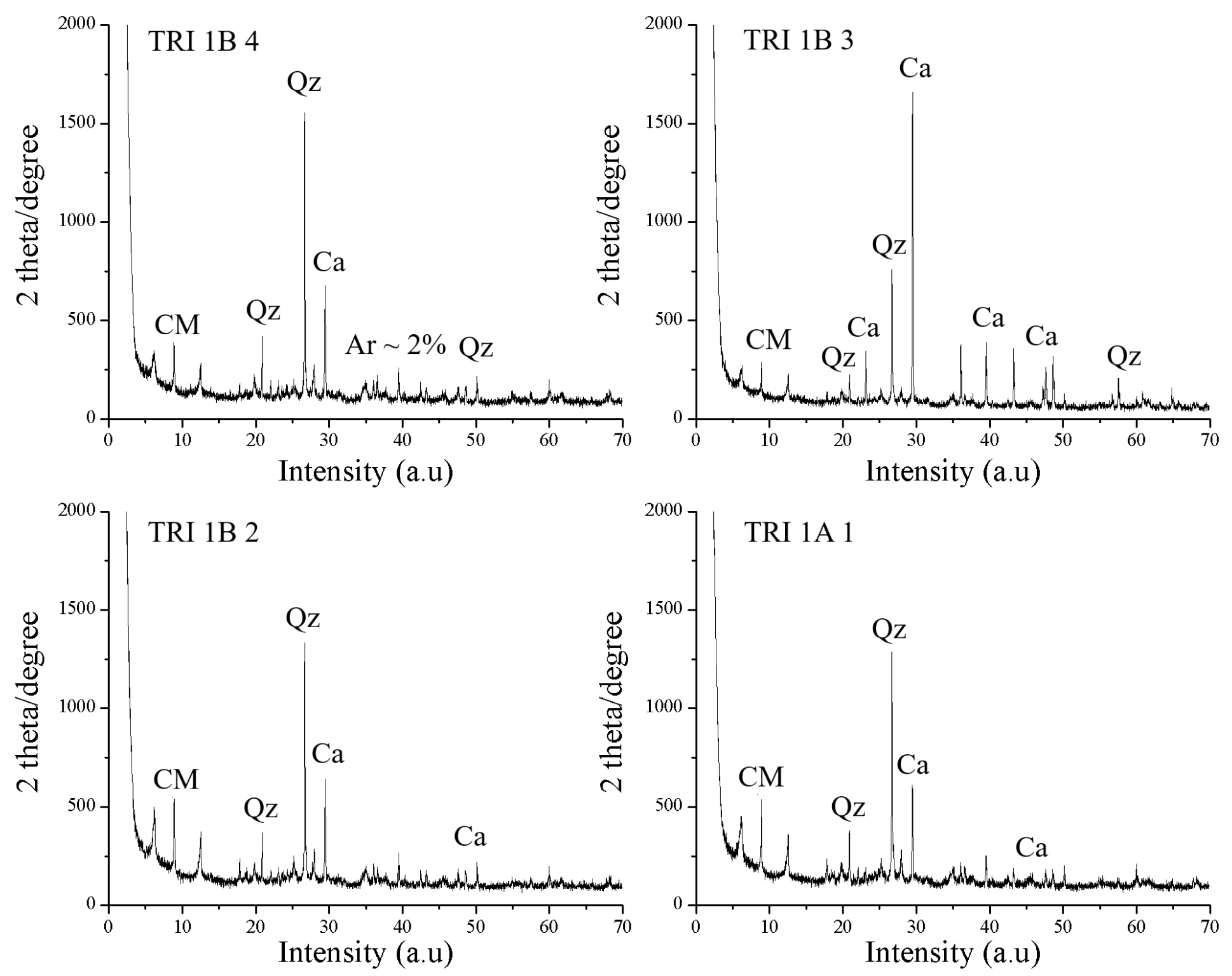

Fig. S4 